\title{
Bandwidth Constrained Multipath Routing Protocol for QoS Provision in MANETs
}

\author{
Priyanka Bhardwaj, Surjeet \\ Department of Electronics \& Communication Engineering, Bharati Vidyapeeth's College of Engineering, \\ New Delhi, India;
}

Priyanka.Bhardwaj@bharatividyapeeth.edu;surjeet.Balhara@bharatividyapeeth.edu

\begin{abstract}
Mobile Ad hoc NETworks (MANETs) are composed of mobile nodes with limited resources and unpredictable node movement. With the on-going evolution in MANETs, provision of Quality of Service (QoS) has become a challenging task. In this paper, a node disjoint Bandwidth Constrained Multipath Routing (BCMR) protocol is proposed which considers bandwidth as the key factor to discover multiple paths for QoS provisioning. BCMR accommodates required bandwidth function in flooding route request packets. Extensive simulation study is carried out to investigate the performance of BCMR. Simulation results reveal that $B C M R$ significantly reduces overheads, minimizes overall end to end delay and significantly improves packet delivery ratio.
\end{abstract}

Keywords: AODV; Bandwidth; MANETs; Multipath; Node-disjoint; QoS; Routing

\section{Introduction}

MANETs consist of several mobile nodes connected by wireless links. MANETs are self-configuring network without having any fixed infrastructure or central administration [1]. Network topology changes continuously due to random mobility of nodes comprising the network [2]. In wired networks, network topology does not frequently change as in MANETs. Due to unpredictable nature of MANETs, mobile nodes may join or leave the network dynamically. Each mobile node in such networks has the capability to communicate directly with other nodes either directly or through multihop communication. MANETs mostly has applications in situations like disaster relief, military operations, rescue operations or conference networking. In these networks, source destination connection may break any time and required to be updated regularly. Quality of Service (QOS) in MANETs is a big challenge. With [95] the evolution of advance technologies in wireless communications, high bandwidth applications such as video or audio streaming, video conferencing and real time data transmission become an important part of application for such networks [3]. These kinds of application require a high level of service quality, so good QoS support is necessary. QoS is a set of service parameters like throughput, overall end-to-end delay, jitter, packet delivery ratio that are essential for any particular application for data transfer through that network [4]. Due to this unpredictable behaviour of MANETs, on demand routing protocols like Dynamic Source Routing (DSR) [5] and Ad hoc On-demand Distance Vector (AODV) [6] are generally implemented. 
Priyanka Bhardwaj, Surjeet; Bandwidth Constrained Multipath Routing Protocol for QoS Provision in MANETS, Transactions on Networks and Communications, Volume 5 No. 3, June (2017); pp: 1-15

These reactive protocols do not require any periodic updates in case of proactive routing protocols like Destination Sequenced Distance Vector (DSDV) [7]. On demand routing protocols discovers a route only when needed by any node to send data to destination. They use a single path routing for each session. Path discovered by these protocols is only path with minimum hop count as no QoS constraints have been put on path discovery.

This paper proposes a node-disjoint Bandwidth Constrained Multipath Routing (BCMR) protocol based on AODV protocol. This approach considers the stability of the route by considering bandwidth constrained multiple paths for data transfer. Only selected route requests are forwarded satisfying QoS requirements. Multiple node-disjoint path discovery technique is used to compute multiple paths.

The rest of the paper is organised as: Section 2 discusses related work in QoS routing and multipath routing. Section 3 discusses stability of multipath with single path approach. Section 4 describes the proposed multipath approach for MANETs. Section 5 gives performance evaluation. Conclusion is summarized in Section 6.

\section{Related Work}

Several routing protocols have been proposed for provision of QoS and supports reliable data transmission over the network [8, 9, 10 and 11]. Basic reactive (on-demand) routing protocols like AODV and DSR have been modified to support multipath routing. Pearlman et al. proposed a modified DSR approach to find node disjoint paths with diversity injection scheme [12]. In this, intermediate nodes can redirect Route Reply (RREP) along multiple paths to the source. Taking AODV as the base protocol, Lee et al. proposed an Ad Hoc On demand Distance Vector-Backup Routing (AODV-BR) protocol [13]. In this approach, multipath is established without creating any extra control message. Back-up routes are provided by overhearing the Route Reply (RREP) messages. Nasipuri et al. proposed two link disjoint paths from source to destination with each intermediate node having one primary path and other as the backup path [14]. Using this approach, life span of the active route is increased. Cho et al. proposed Time Delay On-demand Multipath (TIDOM) routing protocol is proposed [8] which is an extension from Ad hoc OnDemand Multipath Distance Vector (AOMDV) routing protocol [15]. It discovers multiple routes with good energy condition of nodes in route discovery phase. After route request packet arrival at the destination node, sum of residual battery capacity is taken into consideration. Nodes with maximum residual battery capacity are considered for joining the route. Destination will send RREP sequentially for multiple times. Source receives multiple RREP having good residual battery capacity. Simulation results show that network lifetime, packet delivery ratio and energy consumption are much improved in TIDOM as compared to AOMDV. Lai et al. proposed Ad hoc On demand Distance Vector- Adaptive Backup Route (AODV-ABR) protocol in which back-up routes are established taking advantage of RREP overhearing with hop count to the destination [16]. Whenever there is a link failure between nodes in the path, AODV- ABR starts a three-way handshake. Hop count is considered as one of the main factors in route discovery and route maintenance. Control message overheads are large in AODV-ABR like AODV-BR. Ad hoc On demand Distance Vector Base Backup Routing Scheme (AODV-BBS) is proposed by Huang where 'HELLO' message is modified to have a two hop neighbor knowledge [17]. Each mobile node in the network broadcast 'HELLO' messages periodically having information of all neighbours within reach in one hop. The approach uses two hop neighbor's knowledge to discover back-up path during path formation and maintain updates regularly. Anantapalli et al. proposed an analytical model for multipath multihop routing in MANETs [18]. 
Every node in the network is supposed to have some finite buffer. To select a path with minimum delay from different available paths between source and destination nodes, an optimal route strategy has been proposed.

In recent years, much more work is carried out by authors on QoS multipath routing. Yi et al proposed a Multipath Optimized Link State Routing (MP-OLSR) for large and dense networks considering heavy network load and high mobility [19]. Modified Dijkstra algorithm is proposed to find multiple paths from source to destination nodes. This algorithm is modified by using two cost functions to discover multiple or node-disjoint paths. Lee et al. proposed a backup path scheme for MANETs taking AODV as the base protocol [20]. One hop search mechanism is used to discover a backup route for destination. Main QoS parameters considered in this paper are packet delivery ratio and end-to-end delay from source to destination. This approach creates a backup route with a maximum of two hops from restoring node to target node. Performance analysis shows much improvement in terms of packet delivery ratio and endto-end delay characteristics. Jiang et al. proposed Dual Path Node-disjoint Routing (DPNR) protocol considering AODV as the base protocol [21]. The proposed approach work efficiently for data salvation in case of link failure and improves the robustness of available paths. For this it maintains two shortest backup routes from source to destination. This routing protocol performs well in lightly loaded networks as in heavy loaded networks; packet processing, packet collision and channel contention are increased. Kim et al. proposed a multipath routing algorithm for QoS and multimedia services in MANETs based on Ant Colony Optimization (ACO) technique [22]. The proposed algorithm discovers multiple paths by using ACO to provide QoS. Routing packets are adaptively distributed through multiple paths available to the destination to ensure proper network resources utilization. Igartua et al. proposed dynamic Contention Window-Multipath Multimedia Dynamic Source Routing (dCW-MMDSR) [23]. A dynamic Contention Window (dCW) is considered to outperform 802.11 e by assigning smoother values of contention window for each access category. This multipath routing approach is suitable for video streaming depending on the state of the network. Ant Colony Optimization (ACO) technique is used to allocate bandwidth throughout the route [24, 25]. To measure various parameters like delay, bandwidth and next hop availability, ant like agents is used. These are Forward ANT (FANT) and Backward ANT (BANT). Path preference probability is calculated to select a particular path from various available paths. To transmit the data from source to destination, path with highest preference probability is selected. During path discovery, this algorithm finds fault prone nodes and skips them from selected path to satisfy QoS requirements. Performance analysis clearly shows much improvement in packet delivery ratio and throughput. Rajendra Kumar Gupta et al. proposed Node Disjoint Minimum Interference Multipath Routing Protocol (ND-MIM) routing protocol which enables discovery of two node-disjoint minimum interference paths from source to destination [26]. Such paths are identified using HELLO messages. To setup two node-disjoint path, three control messages are used which reduces control overheads. It also helps in setting up backup route faster. Simulation results show significant improvement in overall endto-end delay. Extended AOMDV for Multi-Interface Multi-Channel (EAOMDV-MIMC) and its extended protocol Extended AOMDV for Multi-Interface Multi-Channel with Path Metric (EAOMDV-MIMC-PM) for multi interface multi-channel networks is proposed considering AODV as base protocol [27]. This approach utilizes multiple homogeneous networks interface to improve performance of MANETs. In this, channels are not assigned rather nodes can make use of all available channels. In EAOMDV-MIMC, nodes distribute data packets by estimating channel condition of the network interface queuing. In the extended protocol, estimated channel condition is piggybacked in data packets and propagated further. Ping Dong et al. 
Priyanka Bhardwaj, Surjeet; Bandwidth Constrained Multipath Routing Protocol for QoS Provision in MANETS, Transactions on Networks and Communications, Volume 5 No. 3, June (2017); pp: 1-15

proposed a Beacon-less Geographic Multipath (BGM) routing protocol is proposed that uses location information to discover maximally node-disjoint paths to avoid control messages caused by route discovery [9]. Data packets are forwarded within a disjoint subzone divided by division algorithm. Beaconless mechanism is used in forwarding strategy to reduce number of useless beacons. Zhang et.al proposed an interference based topology control algorithm for delay constrained mobile ad hoc networks. Firstly, minimized the power consumption and at the same time satisfying the interference constraint and then transmission power is increased to meet the delay constraint [28]. MAC protocols are proposed for dynamic channel allocation and cooperative load balancing to improve bandwidth efficiency under non uniform load conditions [29]. CDCA-TRACE algorithm is incorporated to provide support for non uniform load distribution. An opportunistic routing protocol JOKER is proposed to improve the performance of network supporting multimedia traffic as well as enhancing the nodes energy efficiency [30]. New metrics for selecting candidates is developed as packet delivery and distance progress towards the final destination. Ant based multipath backbone routing is proposed for load balancing in MANETs [31]. Source selects the multiple paths with maximum path preference probability using swarm based ant colony optimization technique. Path preference probability is estimated based on next hop availability, delay and bandwidth.

Many QoS aware routing protocols have been proposed based on multipath routing to improve network parameters like throughput, end-to-end delay, jitter, packet delivery ratio. However, the most effective method should improve these parameters as well as should be compatible with the MANETs.

\section{Analysis of stability of multipath with single path approach}

Most of the earlier approach as stated uses single path discovery. In such cases, if a source has data to send and route break occurs in between transmission, then source must reinitiate a route discovery process. This results in significant data loss and delay. Therefore, in many applications where delay and data loss is intolerable, multiple paths are desirable. Multiple paths can be used as an alternate to transfer data without any delay and data loss. Multiple paths also provide fault tolerance. Let us consider a single route $R\left(x_{1}, x_{2}, x_{3} \ldots \ldots . . . x_{n}\right)$. ' $x_{1}^{\prime}$ is source node and ' $x_{n}$ ' is destination node. Let the probability of route break be ' $\mathrm{P}_{\mathrm{br}}$ ' caused by random node movement. Therefore, stability of route ' $\mathrm{P}_{\text {st' }}$ ' can be calculated as

$$
P_{s t}=\left(1-P_{b r}\right) n
$$

So, unstability of the route will be

$$
P_{\text {unst }}=1-\left(1-P_{b r}\right) n
$$

Here $0 \leq 1-\mathrm{Pbr}_{\mathrm{br}} \leq 1$ and $1 \leq \mathrm{a} \leq \mathrm{n}$

Therefore

$0 \leq\left(1-P_{b r}\right) n \leq\left(1-P_{b r}\right) a \leq 1$

$$
\begin{gathered}
\mathrm{P}_{\mathrm{br}}(\mathrm{n}) \leq\left(1-\mathrm{P}_{\mathrm{br}}\right) \mathrm{a} \\
\mathrm{P}_{\mathrm{st}}(\mathrm{n}) \leq \mathrm{P}_{\mathrm{st}}(\mathrm{a})
\end{gathered}
$$

So, it is clear that more the intermediate nodes in a route, route will be more unstable.

Now, taking the case of multiple paths, let us consider two routes $R_{1}\left(y_{1}, y_{2}, y_{3} \ldots \ldots . . . y n\right)$ and $R_{2}\left(z_{1}, z_{2}\right.$, $\left.\mathrm{z}_{3} \ldots \ldots . . . \mathrm{z}_{\mathrm{m}}\right) . \mathrm{P}_{\text {unst }}(\mathrm{i})$ denotes the instability of the two routes with ' $\mathrm{i}$ ' nodes in common. 


$$
\begin{gathered}
P_{\text {unst }}(0)=\left[1-\left(1-P_{b r}\right) n\right]\left[1-\left(1-P_{b r}\right) m\right] \\
P_{\text {unst }}(1)=\left[1-\left(1-P_{b r}\right) n-1\right]\left[1-\left(1-P_{b r}\right) m-1\right]+P_{b r} \\
\text { Punst }(k)=\left[1-\left(1-P_{b r}\right) n-k\right]\left[1-\left(1-P_{b r}\right) m-k\right]+k P_{b r}
\end{gathered}
$$

Let us compare route unstability between Punst(k) and Punst(k-1) as

$P_{\text {unst }}(k)-P_{\text {unst }}(k-1)=\left[1-\left(1-P_{b r}\right) n-k\right]\left[1-\left(1-P_{b r}\right) m-k\right]+k P_{b r}-\left[1-\left(1-P_{b r}\right) n-(k-1)\right]\left[1-\left(1-P_{b r}\right) m-(k-1)\right]+(k-1) P_{b r} \geq$ 0

This clearly shows that

$$
\mathrm{P}_{\text {unst }}(\mathrm{k}) \geq \mathrm{P}_{\text {unst }}(\mathrm{k}-1)
$$

Equation (8) clearly shows that more the common intermediate nodes in a multiple path scenario, worst will be the stability.

\section{QoS Aware Routing}

QoS is a set of mechanisms which tries to utilize various resources provided by the network efficiently to accomplish any particular application. QoS routing provides the required metrics as per the requirement of the application. QoS can be categorized by certain number of network metrics like throughput, end-toend delay, jitter and packet delivery ratio etc. QoS constraint model provides the best possible service as required by the application. In this paper, bandwidth constrained multipath routing has been proposed. A QoS based routing has been proposed that provides feedback about the available bandwidth throughout the route discovery. Multiple routes are selected on the basis of minimum bandwidth requirement as desired by that particular application.

\subsection{Available Bandwidth Estimation}

In bandwidth constrained QoS routing, path is discovered which fulfils the requirement of minimum available bandwidth throughout the route. There are several approaches by which end to end available bandwidth can be calculated. In our approach, end to end available bandwidth is calculated by minimum residual bandwidth among the intermediate nodes throughout the route. As each node shares its available bandwidth between its neighboring nodes, so it is difficult for individual host to calculate residual bandwidth throughout the path. A host will offer bandwidth guaranteed route only if residual bandwidth at a given host is known. However, calculation of residual bandwidth through 802.11MAC is still a difficult problem as the bandwidth is shared among neighbors. Even neighboring hosts are not aware of the traffic status of each other. QoS constrained routing protocol to calculate available bandwidth throughout the route has been proposed by Chen and Heinzelman [32]. Author estimated the residual bandwidth of the host by listening to the channel, the amount of idle time.

Let us consider a network consisting of ' $n$ ' number of mobile nodes i.e. $N_{1}, N_{2}, N_{3}, \ldots \ldots . N_{n}$. Each node in the network will have to maintain two hop neighbor routing tables. Firstly, the one hop neighbor table and then the two-hop neighbor table. Let us consider a mobile node $N_{x}$ with one hop neighbor as $N_{y}$ and two hop neighbor as $\mathrm{N}_{\mathrm{z}}$ as shown in Figure1. 


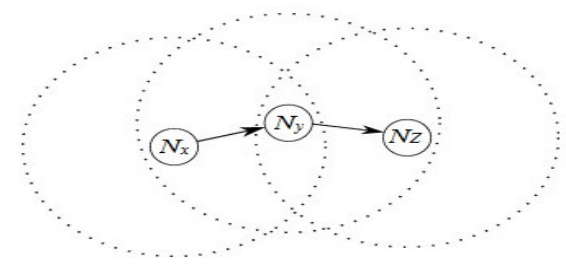

Figure1. $\mathrm{N}_{\mathrm{x}}$ and its two hop neighbors $\mathrm{N}_{\mathrm{y}}, \mathrm{N}_{\mathrm{z}}$

Let consumed bandwidth of $\mathrm{Ny}$ be Bycons and consumed bandwidth $\mathrm{Nz}$ be Bzcons for all inflows and outflows of the data transmission by $N_{y}$ and $N_{z}$. As $N_{y}$ is considered as one hop from $N_{x}$, so there is a direct link from $N_{x}$ to $N_{y}$. Similarly, there is a link directed from $N_{y}$ to $N_{z}$ as $N_{z}$ is two hop neighbor from $N_{x}$. In order to maintain the two-hop neighbor table, these two neighboring host should exchange their one hop table along with their consumed bandwidth periodically via control packet $B_{i c o n s}$. $B_{i c o n s}$ represents consumed bandwidth by node $N_{i}$ i.e. ith node. In this approach, $N_{y}$ and $N_{z}$ have to exchange their routing table along with their consumed bandwidth $\mathrm{B}_{\mathrm{ycons}}$ and $\mathrm{B}_{\mathrm{zcons}}$ respectively.

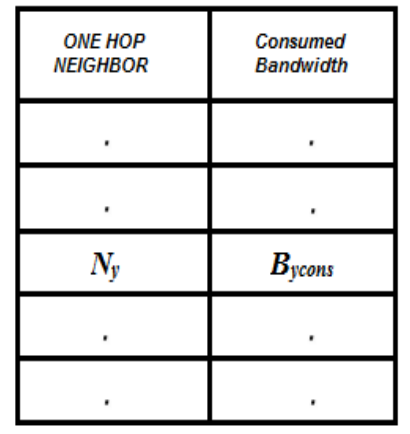

One Hop Neighbor Table of $N_{x}$

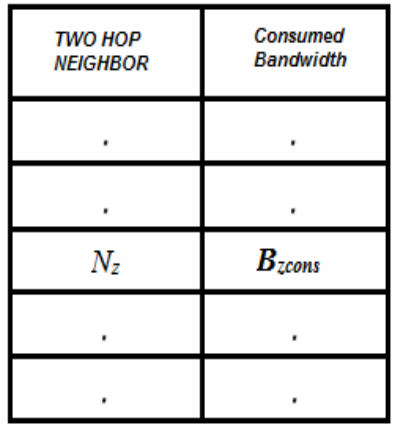

Two Hop Neighbor Table of $N_{x}$

Figure2. Two hop neighbor table of node $\mathrm{N}_{\mathrm{x}}$

When $\mathrm{N}_{\mathrm{y}}$ and $\mathrm{N}_{\mathrm{z}}$ receives Bicons from $\mathrm{N}_{\mathrm{x}}$ node, these nodes will reply with their consumed bandwidth $B_{y c o n s}$ and $B_{z c o n s}$ respectively. Two hop routing table of node $N_{x}$ is shown in Figure 2 . When $N_{x}$ receives the reply about currently consumed bandwidth $\mathrm{B}_{\text {ycons }}$ and $\mathrm{B}_{\text {zcons }}$ from both its two hop neighbors $\mathrm{N}_{\mathrm{y}}$ and $\mathrm{N}_{z}$ respectively, the residual bandwidth is easily calculated by subtracting consumed bandwidth of the two neighboring hops from maximum available bandwidth.

$$
\begin{gathered}
n \\
B_{\text {residual }}=\left(B_{\max }-\sum B_{\text {icons }}\right) / W_{f} \\
i=0
\end{gathered}
$$

$B_{\text {residual }}$ represents the available residual bandwidth, $B_{\max }$ is maximum available bandwidth across a path and $\mathrm{B}_{\text {icons }}$ is bandwidth used by $\mathrm{Ni}$. $\mathrm{W}_{\mathrm{f}}$ denotes the weight factor as defined below in equation (2). The division of the residual bandwidth by the weight factor $W_{f}$ is done due to 802.11MAC. The control messages like RTS, CTS and ACK are induced by MAC. These control messages also consume some 
bandwidth, that's why back off scheme is not successful for use of the entire bandwidth and also, collision of packets can be there.

The weight factor $\mathrm{W}_{\mathrm{f}}$ can be calculated as

$$
W_{f}=\{R T S+C T S+(\text { Data }+ \text { MAChdr }+ \text { IPhdr })\} / \text { Data }
$$

All the terms like RTS, CTS.... are used to represent the size of these packets respectively. The value of the weight factor calculated above is more as fading errors can cause the retransmission of control or data packets.

\subsection{Proposed Route Discovery}

Let us consider a dense network of mobile nodes having same transmission range. The base protocol considered for our proposed route discovery is AODV. In this protocol, each mobile node has its own routing table based on reactive routing. Each node adds or updates a new route in a reactive manner. If a node has data to transmit to a destination, firstly it will check whether there is any route available in its routing table. If the source finds a route to destination, it can start transmission of data directly. If there is no route for that destination, the source node has to initiate a route discovery process. A Route Request (RREQ) packet is generated at source node and broadcasted in the network to find route to the destination. Each neighbor node that gets RREQ adds or updates its RREQ table and broadcasts the packet again. Intermediate nodes discard late RREQ packets that have same source node and destination sequence number. After receiving the RREQ packet, destination node replies with a Route Reply (RREP) packet to the source node through symmetric links. As source node receives RREP, the route is set up and source node starts transmission of data to the destination.

\subsubsection{Implementing QoS in Route Discovery}

Our approach is based on bandwidth constrained multipath node disjoint route discovery and fast route recovery. Node disjoint paths are those paths, which have only source and destination node common. No intermediate node is common in node disjoint paths as shown in Figure 3.

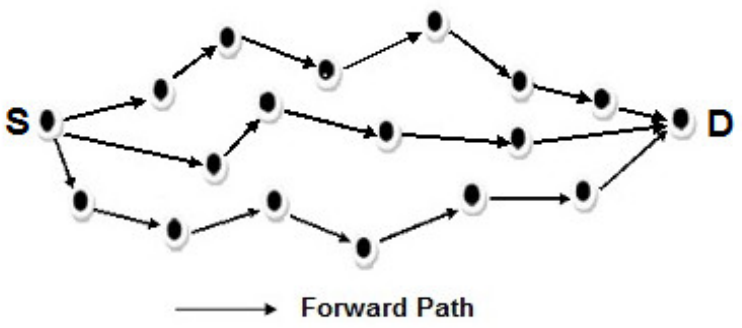

Figure 3. Node disjoint paths

The main advantage of node disjoint paths is that they are independent of each other. If any route break is there, data session can be resumed through one of the alternate multi paths available. To discover such paths, AODV RREQ header is modified. AODV control packet for route discovery process consist of request identifier, source address, destination address, destination sequence number, prefix size, hop count and lifetime of the route [6]. To initiate the QoS route discovery RREQ header has been changed to (REQ BW, AODV RREQ Header). REQ BW indicates required bandwidth to satisfy service requirements desired by the application. If the residual bandwidth on that link is greater than required bandwidth, it will forward 
the RREQ. Otherwise RREQ will be discarded by that node. After receiving RREQ, the whole procedure of host is shown in Figure 4.

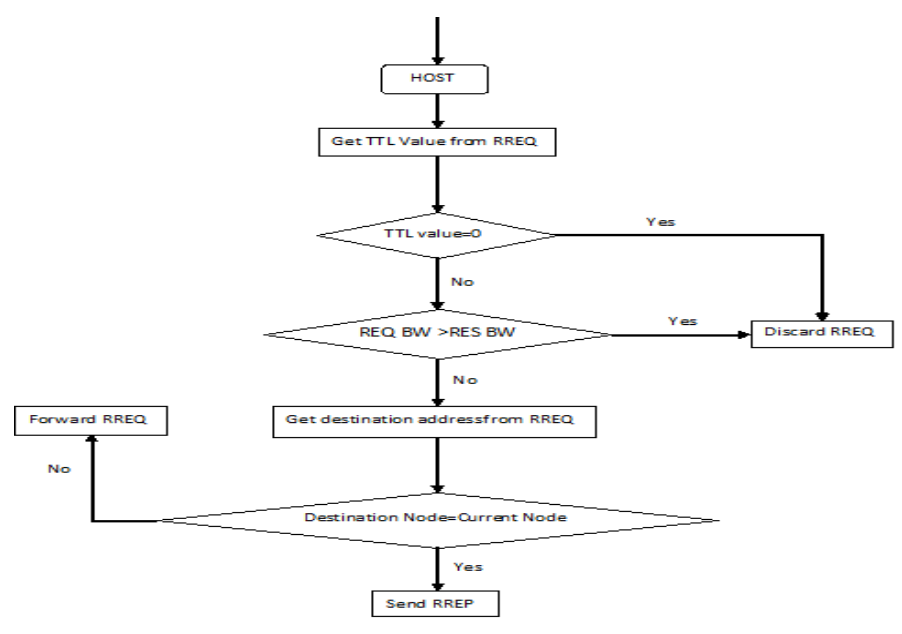

Figure 4. Host's working procedure

When any intermediate node receives RREQ packet, it first examines to see duplicate RREQ with same source node and destination sequence number to discover node disjoint paths. If duplicate RREQ is received by the intermediate node, RREQ will be discarded. Only the first received RREQ packet will be forwarded When RREQ packed is received by the destination node, the node sends a RREP. Each intermediate node forwards RREP to source node through symmetric links. As source node receives the RREP packet, it adds route in its routing table.

\subsection{Proposed Route Reply}

When a RREQ packet is received by the destination node, the node sends a RREP to the source. RREP follows the reverse path as stored in the RREQ received table in intermediate nodes. RREP packet in BCMR is modified with three more fields like (Node seq, Reply source, Path no., AODV RREP Packet). Node seq stores the list of all intermediate nodes through which RREP has traversed so far. Reply source field contains the address of the node from which RREP packet has been generated. Node seq and Reply source are added to avoid routing loops and to discover node disjoint paths. Path no. gives the number of path in sequence as received by the source node. When first RREP arrives at source node, source node stores the route in its routing table with Path no.1 and so on.

\subsection{Proposed Route Maintenance}

In MANETs, route link breaks frequently due to uncertain node mobility and packet collision. In conventional AODV whenever there is a route break during data transmission, it simply sends a Route Error (RERR) packet to its upstream node. After arrival of RERR packet at source node it initiates a new route discovery. In BCMR, when a node detects a route break, it will perform one hop route recovery process for smooth transmission of data. In this process, when a node detects a route break, it will send Immediate Route Request (IRREQ) to its one hop neighbours to transmit the data through alternate route. If any node in one hop neighbour have route to destination, it will send Immediate Route Reply (IRREP) to the node and data will be transmitted through this alternate route. If there is no such route available, immediate upstream node will send a RERR to the upstream nodes. When RERR packet arrives at source 
node, source node invalidates this route related to that broken link and relays the data to alternate route available to the destination.

\section{Performance Evaluation}

Initially mobility of the nodes is varied as $0 \mathrm{~m} / \mathrm{s}, 5 \mathrm{~m} / \mathrm{s}, 10 \mathrm{~m} / \mathrm{s}, 15 \mathrm{~m} / \mathrm{s}, 20 \mathrm{~m} / \mathrm{s}$ and $25 \mathrm{~m} / \mathrm{s}$. Number of nodes taken for simulation are 30 nodes and 50 nodes with different mobility scenario. Weight factor considered for BCMR and CAAODV (L) [32] is 1.2.

\subsection{Performance Metrics}

To compare the performance of our proposed approach, following performance metrics are considered.

Average end-to-end delay gives the average time delay that a data packet has consumed from the time it was sent by the source to the time delivered at the destination.

Average packet delivery ratio is the ratio of number of data packets received by the destination to the number of data packets actually sent by the source.

Normalized control overhead is the number of control packets sent to the network hop-wise to the number of data packets delivered at the destination.

\subsection{Simulation Environment}

The covered area for simulation environment is $1000 \mathrm{~m} \times 1000 \mathrm{~m}$. Different nodes communicate via radio signals having transmission range of $250 \mathrm{~m}$. Channel bandwidth taken is $2 \mathrm{Mbps}$. In our simulation, IEEE 802.11 is used as MAC layer protocol. Initially nodes are randomly distributed in the simulation area. Random waypoint mobility model determines the mobility of the nodes. Path loss model is Two Ray Ground model. For Constant Bit Rate (CBR) data sessions, node pairs are randomly selected with each CBR session generating 5 packets per second with 512 bytes as each data packet size. Table 1 gives the list of simulation parameters used for analysis of our proposed approach.

Table1. Simulation Parameters

\begin{tabular}{|l|l|}
\hline PARAMETER & \multicolumn{1}{c|}{ VALUE } \\
\hline Simulation Area & $1000 \mathrm{~m} \times 1000 \mathrm{~m}$ \\
\hline Number of nodes & 30,50 \\
\hline Mobility model & Random waypoint model \\
\hline Mobility & $0-25 \mathrm{~m} / \mathrm{sec}$ \\
\hline Path loss model & Two ray ground \\
\hline Channel bandwidth & $2 \mathrm{Mbps}$ \\
\hline Transmission range & $250 \mathrm{~m}$ \\
\hline Packet size & 512 bytes \\
\hline MAC protocol & IEEE $802.11 \mathrm{DCF}$ \\
\hline Simulation time & $100 \mathrm{~s}$ \\
\hline CBR data sessions & 10 \\
\hline
\end{tabular}


To analyze the performance of our proposed routing protocol with different weight factors and compare with conventional AODV, CAAODV (H) [32] and CAAODV (L) [32]. Basically, two parameters are varied in our simulation scenario.

(i)Varying mobility of the nodes

(ii)Varying number of mobile nodes

For performance evaluation of our proposed BCMR scheme, velocity of the mobile nodes is varied in the simulation from $0 \mathrm{~m} / \mathrm{s}$ to $25 \mathrm{~m} / \mathrm{s}$. Number of mobile nodes taken in our simulation is 30 nodes and 50 nodes.

\subsection{Results and Discussion}

Initially mobility of the nodes is varied as $0 \mathrm{~m} / \mathrm{s}, 5 \mathrm{~m} / \mathrm{s}, 10 \mathrm{~m} / \mathrm{s}, 15 \mathrm{~m} / \mathrm{s}, 20 \mathrm{~m} / \mathrm{s}$ and $25 \mathrm{~m} / \mathrm{s}$. Number of nodes taken for simulation are 30 nodes and 50 nodes with different mobility.

\section{Average end-to-end delay}

Figure 5 and Figure 6 shows the plot of end-to-end delay vs. node mobility using the simulation parameters given in Table 5.1 with different number of nodes i.e. 30 nodes and 50 nodes respectively. From the comparison, we can observe that average end-to-end delay in our proposed approach is much less as compared with CAAODV (H), CAAODV (L) [31] and AODV with different number of nodes and mobility. Comparative results of end-to-end delay of our proposed node-disjoint scheme and other proposed schemes are shown in Figure 5 and Figure 6. Results show improvement in end-to-end delay with weight factor as 1.2 for BCMR and CAAODV $(\mathrm{L})$ as shown. In proposed approach, end-to-end delay is minimized due to availability of alternate route for the destination. This eliminates the route rediscovery latency due to active route failure. As per the simulation results shown in Figure 5.5 and Figure 5.6, average of $32 \%$ and $36 \%$ improvement in terms of end-to-end delay is obtained for BCMR respectively as compared to AODV considering 30 and 50 nodes in the network. As compared to CAAODV (H) and CAAODV (L), we have obtained improvement of $9 \%$ and $10 \%$ respectively in BCMR for 30 nodes and in case of 50 nodes; we have obtained improvement of $11 \%$ and $13 \%$ respectively in BCMR.

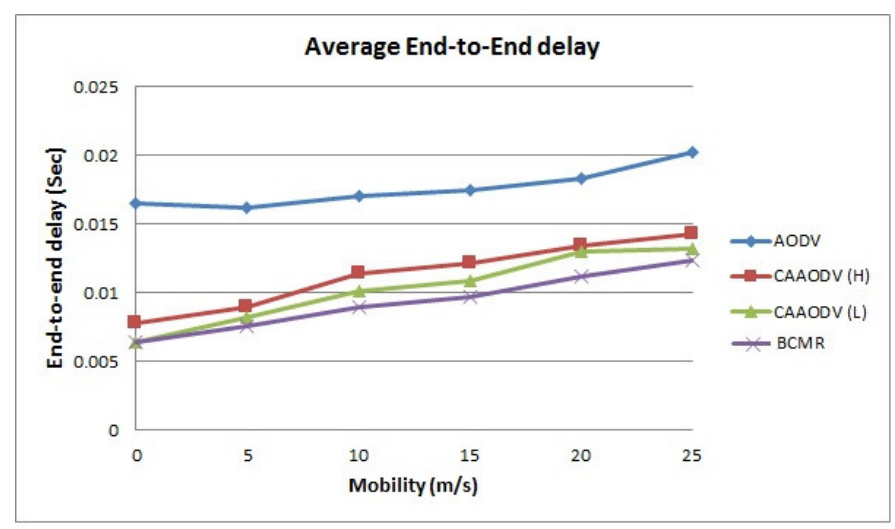

Figure 5. Average End-to-End Delay v/s Node mobility (30 nodes) 


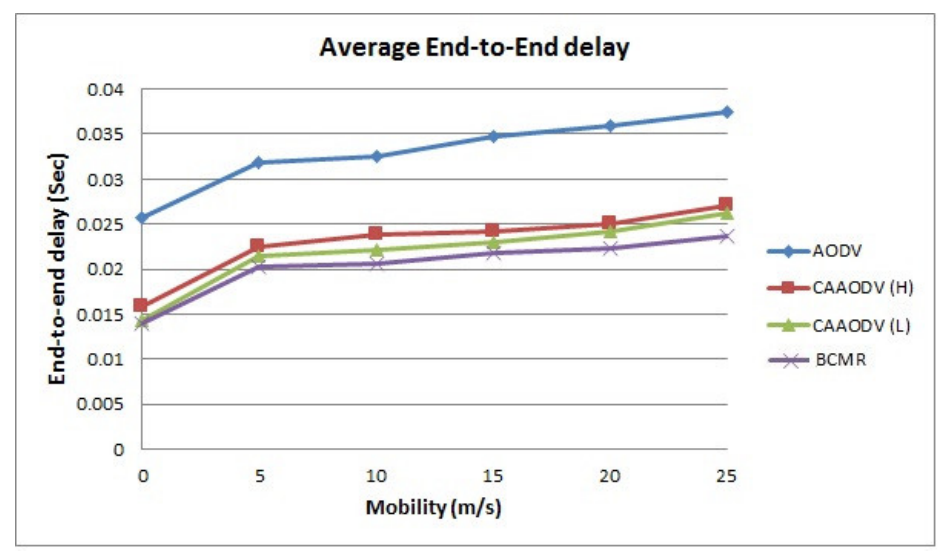

Figure 6. Average End-to-End Delay v/s Node mobility (50 nodes)

\section{Normalized control overhead}

From Figure 7 and Figure 8 shows the plot of normalized control overhead vs. node mobility using the simulation parameters given in Table 1 with different number of nodes i.e. 30 nodes and 50 nodes respectively. From the comparison, we can observe that control overheads in our proposed approach is much less as compared with CAAODV $(\mathrm{H})$, CAAODV $(\mathrm{L})$ and AODV with different number of nodes and mobility. Results show improvement in control overheads with weight factor as 1.2 for BCMR and CAAODV (L) as shown. In proposed approach, control overheads are minimized due to bandwidth constrained routing for the destination. As per the simulation results shown in Figure 7 and Figure 8, average of 23\% and $26 \%$ improvement in terms of control message overheads is obtained for BCMR respectively as compared to AODV considering 30 and 50 nodes in the network. As compared to CAAODV $(\mathrm{H})$ and CAAODV (L), we have obtained improvement of $8 \%$ and $9 \%$ respectively in BCMR for 30 nodes in terms of control message overheads. Taking 50 nodes, we have obtained improvement of $10 \%$ and $12 \%$ respectively in BCMR as compared to CAAODV $(\mathrm{H})$ and CAAODV (Listen).

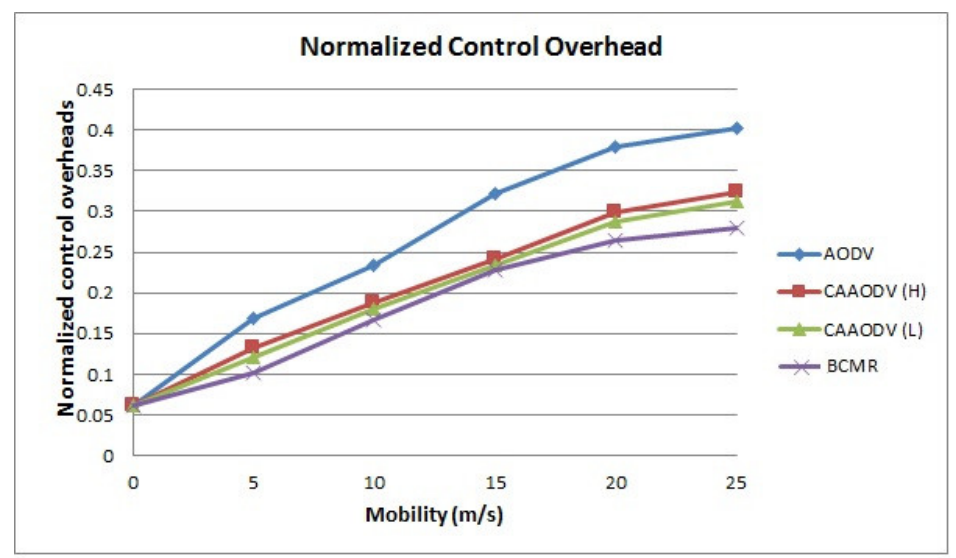

Figure 7. Normalized Control Overhead v/s Node Mobility (30 nodes) 


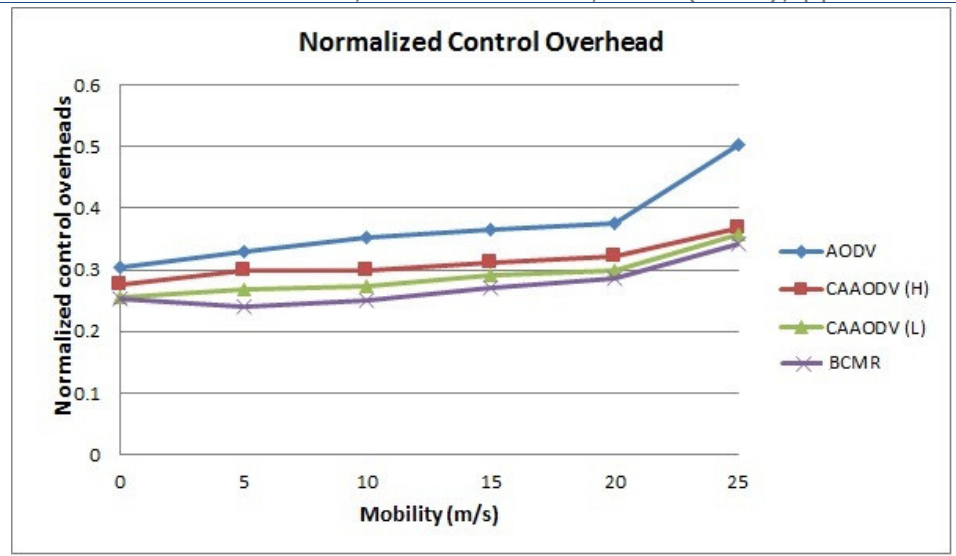

Figure 8. Normalized Control Overhead v/s Node Mobility (50 nodes)

\section{Average packet delivery ratio}

From Figure 9 and Figure 10 shows the plot of packet delivery ratio vs. node mobility using the simulation parameters given in Table 5.1 with different number of nodes i.e. 30 nodes and 50 nodes respectively. From the comparison, we can observe that packet delivery ratio in our proposed approach is significantly improved as compared with CAAODV admission scheme (Hello), CAAODV (Listen) and AODV with different number of nodes and mobility. Results show improvement in packet delivery ratio with weight factor as 1.2 for BCMR and CAAODV (Listen) as shown. In proposed approach packet delivery ratio is improved due to availability of alternate route for the destination. This minimizes packet loss during transmission of data. As per the simulation results shown in Figure 9 and Figure 10, average of 12\% and 14\% improvement in terms of packet deliver ratio is obtained with 1.2 as weight factor for BCMR respectively as compared to AODV considering 30 and 50 nodes in the network. As compared to CAAODV $(\mathrm{H})$ and CAAODV $(\mathrm{L})$, we have obtained improvement of $6 \%$ and $5 \%$ respectively in BCMR for 30 nodes and improvement of $7 \%$ and $5 \%$ respectively in BCMR for 50 nodes in terms of packet delivery ratio.

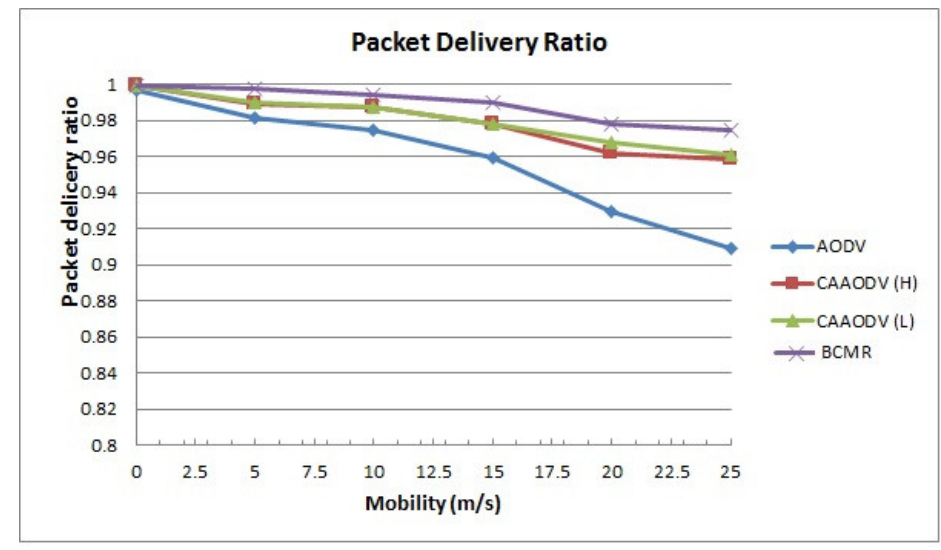

Figure 9: Packet Delivery Ratio v/s Node Mobility (30 nodes) 


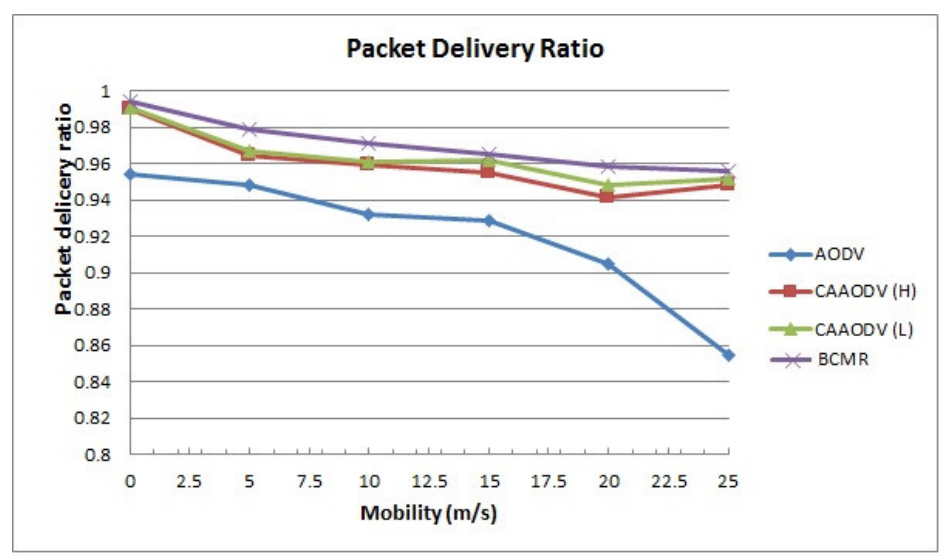

Figure 10: Packet Delivery Ratio v/s Node Mobility (50 nodes)

\section{Conclusion}

This paper proposes a bandwidth constrained multipath routing protocol for QoS provisioning. The use of node-disjoint multiple paths minimizes frequent route recovery and route rediscovery due to frequent route failures as in conventional AODV. Simulation results have shown significant improvements in terms of certain QoS parameters like end-to-end delay, control overheads and packet delivery ratio for different node mobility.

QoS is the most important issue for latest computer networks. As MANETs follows a distributed and uncertain environment, prioritized QoS is more suitable for such networks. Frequent link failure is major issue in MANETs, therefore alternate route strategies should be implemented as per QoS requirements.

\section{REFERENCES}

[1]. Perkins, C. E., Ad Hoc Networking- An Introduction, Ad Hoc Networking, 2001: p 1-28.

[2]. Abolhasan, M., et al., A review of routing protocols for mobile ad hoc networks, Elsevier Journal of Ad hoc Networks, 2004. 2( 1): p. 1-22.

[3]. Chen, S. and K. Nahrstedt, K., An overview of quality of service routing for next-generation high-speed networks: problems and solutions, IEEE Network: The magazine of Global Internetworking, 1998. 12(6): p. 64-79.

[4]. Chakrabarti, S., QoS issues in ad hoc wireless networks, IEEE Communication Magzine, 2001. 39(2): p.142148.

[5]. Johnson, D. B., et al., DSR: The Dynamic Source Routing Protocol for Multi-Hop Wireless Ad Hoc Networks, Ad Hoc Networking, 2001. 5: p. 139-172.

[6]. Perkins, C. E., et al., Ad Hoc On Demand Distance Vector (AODV) Routing, RFC 3561, 2003.

[7]. Perkins, C. E. and P. Bhagwat, Highly dynamic destination-sequenced distance-vector routing (DSDV) for mobile computers, Proceedings of the ACM SIGCOMM, 1994. 24(4): p. 234-244. 
Priyanka Bhardwaj, Surjeet; Bandwidth Constrained Multipath Routing Protocol for QoS Provision in MANETS, Transactions on Networks and Communications, Volume 5 No. 3, June (2017); pp: 1-15

[8]. Cho, W., et al., Time Delay On-demand Multipath Routing Protocol in Mobile Ad-hoc Networks, IEEE International Conference on Ubiquitous and Future Network, 2011.

[9]. Dong, P., et al., A Beacon-Less Geographic Multipath Routing Protocol for Ad Hoc Networks, Mobile Networks and Applications, 2013. 18(4): p 500-512.

[10]. Krishna, P. V., et al., Quality-of-service enabled ant colony-based multipath routing for mobile ad hoc networks, IET Communications, 2012.6(1): p. 76-83.

[11]. Surjeet, et al., QoS Bandwidth Estimation Scheme for Delay Sensitive Applications in MANETs, Communications \& Networks, 2013. 5(1): p. 1-8.

[12]. Pearlman, M.R., et al., On the impact of alternate path routing for load balancing in mobile ad hoc networks, Proceedings of ACM Mobile Ad Hoc Networking and Computing, 2000: p. 3-10.

[13]. Lee, S.J. and Gerla, M., AODV-BR: Backup routing in Ad Hoc networks, Proceedings of IEEE Wireless Communications and Networking Conference, 2000. 3: p. 1311-1316.

[14]. Nasipuri, A., et al., Performance of multipath routing for on-demand protocols in ad hoc networks, ACM/Kluwer Mobile Networks and Applications Journal, 2001. 6(4): p. 339-349.

[15]. Marina, M. K. and Das, S. R., Ad hoc on-demand multipath distance vector routing, Wiley Wireless Communications and Mobile Computing, 2006. 6(7): pp. 969-988.

[16]. Lai, W. K., et al., Adaptive backup routing for ad-hoc networks, Computer Communications, 2007. 30(2): p. 453-464.

[17]. Huang, T., et al., AODV-Based Backup Routing Scheme in Mobile Ad Hoc Networks, International conference on Communications and Mobile Computing, 2010. 3: p. 254-258.

[18]. Anantapalli, M. K. and Li, W., Multipath multihop routing analysis in mobile ad hoc networks, Wireless Networks, 2010. 16(10): p 79-94.

[19]. Yi, J., Multipath optimized link state routing for mobile ad hoc networks, Ad Hoc Networks, 2011. 9(1): $p$ 28-47.

[20]. Lee, K., A backup path routing for guaranteeing bandwidth in mobile ad hoc networks for multimedia applications, Journal of Multimedia Tools and Applications, 2012. 57(2): p.439-451.

[21]. Jiang, F., et al., Dual path node-disjoint routing for data salvation in mobile ad hoc, The Journal of Supercomputing, 2012. 59(1): p. 268-296.

[22]. Kim, S., An Ant-based Multipath Routing Algorithm for QoS Aware Mobile Ad-hoc Networks, Wireless Personal Communications, 2012. 66(4): p. 739-749.

[23]. Igartua, M.A., et al., Dynamic framework with adaptive contention window and multipath routing for video-streaming services over mobile ad hoc networks, Telecommunication Systems, 2012. 49(4): p. 379390. 
[24]. Caro, G. D., Ant colony optimization and its application to adaptive routing in telecommunication networks, PhD thesis, University Libre de Bruxelles, Brussels, Belgium, 2004.

[25]. Gunes, M., et al., ARA-the ant colony based routing algorithm foe MANETs, Proceedings of International Conference on Parallel Processing Workshops, 2002: p.79-85.

[26]. Gupta, R. K., Node Disjoint Minimum Interference Multipath (ND-MIM) Routing Protocol for Mobile Ad hoc Networks, International Journal of Advanced Research in Computer Science and Software Engineering, 2012. 2(3): p. 128-131.

[27]. Kok, G., et al., EAOMDV-MIMC: A Multipath Routing Protocol for Multi-Interface Multi-Channel Mobile Ad-Hoc Networks, Wireless Network Communications, 2013. 73(3): p. 1-30.

[28]. Zhang, X. M., et al., Interference-based Topology Control Algorithm for Delay-constrained Mobile Ad hoc Networks, IEEE Transactions on Mobile Computing, 2015. 14(4): p. 742-753.

[29]. Karaoglu, B. and Heinzelman, W., Cooperative Load Balancing and Dynamic Channel Allocation for ClusterBased Mobile Ad Hoc Networks, IEEE Transactions on Mobile Computing, 2015. 14(5): p.-951-963.

[30]. Iborra, R. and Cano, M., JOKER: A Novel Opportunistic Routing Protocol", IEEE Journal on Selected Areas in Communications, 2016. 34(5): p-1690-1703.

[31]. Selvi, P. F. A. and Manikandan, M., Ant based multipath backbone routing for load balancing in MANET, IET Communications, 2017. 11(1): p-136-141.

[32]. Chen, L. and Heinzelman, W., QoS-Aware Routing Based on Bandwidth Estimation for Mobile Ad Hoc Networks, IEEE Journal on Selected Areas in Communications, 2005. 23(3): p. 561-572. 